\title{
Aplicativo Exatas: Ferramenta de apoio ao aprendizado móvel
}

\author{
Miguel Ângelo Rodrigues Mocbel ${ }^{1}$, Fabricio de Souza Farias ${ }^{1}$
}

\author{
'Faculdade de Sistemas de Informação - Universidade Federal do Pará (UFPA) \\ 68400-000 - Cametá - PA - Brazil \\ \{miguelangelo, fabricosf\}@ufpa.br
}

\begin{abstract}
Resumo. Cada vez mais, alunos buscam informações complementares na Internet para reforçar seus conhecimentos acerca de determinado assunto. Diante deste contexto, neste trabalho é apresentado o cenário de uso, desenvolvimento e implementação do software Exatas. Este é baseado em mobile-learning e visa auxiliar estudantes do ensino médio que pretendem utilizar ferramentas tecnológicas para auxiliar em seu processo de aprendizagem. Para validação, o Exatas foi sujeito a avaliação por alunos do ensino superior e por usuários da loja virtual de aplicativos. Os resultados obtidos demonstram altos índices de aceitação por parte dos usuários e destacam o potencial da ferramenta.
\end{abstract}

\section{Cenário de uso}

O estudante de ensino médio do século XXI que tem interesse em ingressar como aluno em uma Universidade pública ou privada é submetido na maioria dos casos ao ensino tradicional baseado em aulas expositivas que visam a preparação para o Exame Nacional do Ensino Médio (ENEM). Durante o ensino tradicional, o professor explica os conteúdos usando os recursos tecnológicos disponíveis em cada instituição como: quadro branco, caneta para quadro branco, projetor multimídia e computador. Para além da sala de aula, o aluno pode também se tornar agente ativo do seu aprendizado buscando se beneficiar de conteúdos, exercícios e vídeos disponíveis na Internet, os quais podem funcionar como complementos da formação em sala de aula. O complemento do conhecimento fora da sala de aula é uma alternativa necessária de formação, pois de acordo com Arantes e Seabra (2016), alunos que desejam cursar uma universidade enfrentam uma grande concorrência, e por isso, precisam passar por um processo de preparação longo e intenso.

A Internet tem se consolidado como um caminho para o conhecimento, já que é possível buscar e acessar diversos conteúdos de forma independente, através de computadores e smartphones. A popularidade de smartphones tem criado um cenário de aplicação para o ramo educacional. A esse meio de estudo mediado por tecnologias móveis, chama-se de aprendizagem com mobilidade ou Mobile-Learning (m-learning). Segundo Crompton (2013), m-learning é um aprendizado realizado através de vários contextos por meio de interação social e de conteúdo a partir de dispositivos de tecnologia móvel.

Dentre as ferramentas de tecnologias móveis, os aplicativos têm ganhado popularidade em diversas categorias. Na loja virtual da Google, Google Play ${ }^{l}$, a categoria educação detêm o maior número de aplicativos, muitos desses aplicativos abordam

' https://play.google.com/store 
conteúdos de ensino médio e podem auxiliar na preparação para o ENEM. Em relação as pesquisas acadêmicas, Sena et al. (2014) apresentou um levantamento bibliográfico acerca dos principais aplicativos baseados em m-learning. Como resultado, os autores concluíram que há a necessidade do desenvolvimento de um aplicativo de apoio a aprendizagem de matemática. Conforme os autores, os recursos de quiz (exercícios), vídeos (videoaulas), gráficos (desempenho) e conteúdo textual são necessários para atendimento efetivo de demandas dos alunos.

Embora existam esforços que visam apresentar soluções baseadas em m-learning como ferramenta auxiliar ao processo de aprendizagem de alunos do ensino médio, muitas propostas são voltadas para uma área de conhecimento específica, por exemplo, aplicações que envolvem somente matemática [Arantes e Seabra 2016], somente física [Perez, Viali, e Lahm 2015] ou somente química [Mendes e Junior 2017] [Farias et al. 2017], isto é, não abordando um aspecto genérico em relação ao conteúdo, e também não levando em consideração uma organização que disponibilize conteúdos textuais, listas de exercícios e videoaulas em uma mesma plataforma de ensino-aprendizagem. Diante deste contexto, este trabalho apresenta o software Exatas. O Exatas é um aplicativo composto por diversas matérias do ensino médio compreendidas nas disciplinas de física, química e matemática. Além disso, em consonância com Sena et al. (2014) e de acordo com o melhor do nosso conhecimento, esta é a primeira ferramenta que apresenta aos seus usuários de forma organizada, conteúdos teóricos, listas de exercícios, desempenho e videoaulas explicativas.

\section{Desenvolvimento}

O processo de desenvolvimento do software Exatas foi dividido em três etapas. Durante a primeira etapa, foi realizada uma analise acerca dos aplicativos educacionais através de uma revisão da literatura e busca pelos principais softwares disponíveis para plataformas móveis. Como resultado da analise, foram definidas as principais funcionalidades voltadas para o preenchimento de lacunas que aplicativos já disponibilizados possuem.

$\mathrm{Na}$ segunda etapa, as funcionalidades foram modeladas em diagrama de caso de uso e modelo de entidade-relacionamento. O diagrama de caso de uso produzido é composto por 01 ator Aluno e 16 casos de uso, enquanto que, o modelo de entidaderelacionamento é formado por 7 entidades/classes e 10 relacionamentos. E na terceira etapa, o software foi implementado para plataforma Android ${ }^{2}$ utilizando a linguagem de programação $\mathrm{Java}^{3}$, através do ambiente de desenvolvimento integrado (IDE) Android Studio $^{4}$. Para armazenamento e comunicação das informações, foram utilizados servidores de banco de dados e web, respectivamente, $M y S Q L^{5}$ e Apache Web Server ${ }^{6}$, hospedados no serviço de computação em nuvem da Amazon $^{7}$. No projeto de banco de dados, foi utilizada linguagem SQL, enquanto que no servidor web, foi utilizado a

\footnotetext{
https://www.android.com/

${ }^{3}$ https://www.java.com/pt_BR/about/whatis_java.jsp

' https://developer.android.com/studio/

s https://www.oracle.com/br/mysql/index.html

${ }^{6}$ https://httpd.apache.org/

https://aws.amazon.com/pt/
} 
VII Congresso Brasileiro de Informática na Educação (CBIE 2018)

Anais dos Workshops do VII Congresso Brasileiro de Informática na Educação (WCBIE 2018)

linguagem de programação $P H P^{8}$ para garantir a transmissão de informação entre o aplicativo e os servidores. A Figura 1 ilustra a estrutura geral de funcionamento do Exatas. Pode-se observar que no lado do usuário há o aplicativo que apresenta os conteúdos de forma híbrida, ou seja, conteúdo em texto e desempenho disponíveis de forma offline, e questões e vídeos disponíveis de forma online, o que reduz o espaço de armazenamento necessário para instalação, assim como remove a necessidade de atualização do software em caso de disponibilidade de novas questões ou vídeos. Para disposição offline do conteúdo e desempenho, é implementado um banco de dados interno durante a instalação do software. Já para o lado dos servidores, são apresentados os servidores de banco de dados e web. O servidor de banco de dados é responsável em armazenar os dados dos usuários, questões cadastradas e links de vídeos. Por fim, o servidor web é responsável em manter e gerenciar a comunicação das informações entre a aplicação para plataforma móvel e o banco de dados em nuvem.

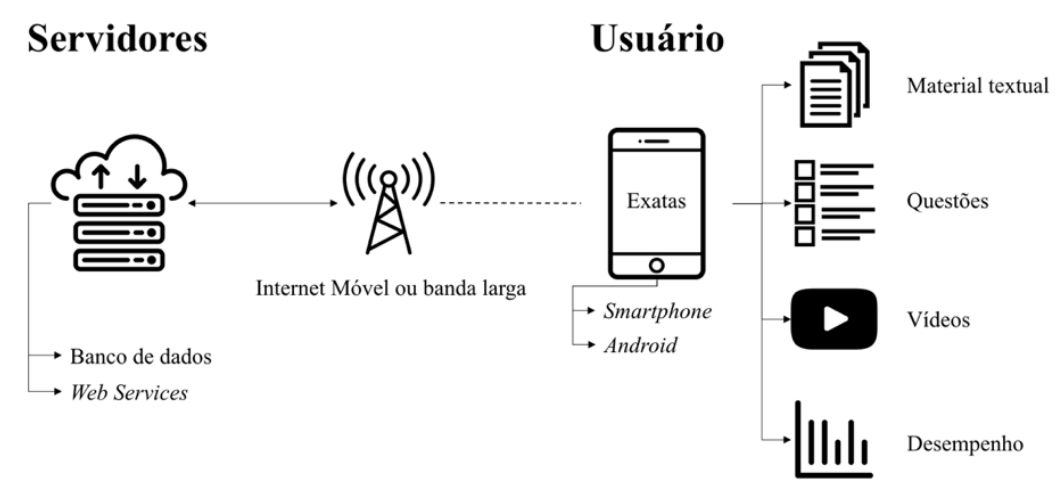

Figura 1 - Esquema geral de funcionamento do Exatas.

Após o cumprimento das etapas de desenvolvimento, o software Exatas foi validado por alunos dos cursos de pedagogia e matemática da Universidade Federal do Pará (UFPA), e por usuários da plataforma Android. A avaliação por alunos de graduação ocorreu em novembro de 2015, enquanto que, a validação por usuários do Google Play ocorreu de dezembro de 2015 até setembro de 2018.

\section{Apresentação do Software}

O Exatas (https://play.google.com/store/apps/details?id=br.ufpa.labex.exatas) é um software gratuito que objetiva a simplicidade de operação para o usuário, logo o acesso a seu conteúdo é permitido de forma facilitada, isto é, apenas exigindo a criação de uma conta no aplicativo. Na Figura 2 (a) é exibida a tela de inicialização do aplicativo, enquanto que as Figuras 2 (b) e 2 (c) exibem as telas de login e criação de conta, respectivamente.

$\mathrm{Na}$ inicialização é apresentada a logo do aplicativo e do laboratório responsável. Já na tela de login, o usuário pode adicionar seus dados para acessar sua conta, assim como, pode selecionar a opção para recuperar sua senha. Enquanto que, na aba de criação de conta, o usuário pode selecionar seu perfil entre as opções de aluno e professor, assim como, registrar-se no sistema.

${ }^{8}$ https://secure.php.net/manual/pt_BR/intro-whatis.php 
VII Congresso Brasileiro de Informática na Educação (CBIE 2018)

Anais dos Workshops do VII Congresso Brasileiro de Informática na Educação (WCBIE 2018)

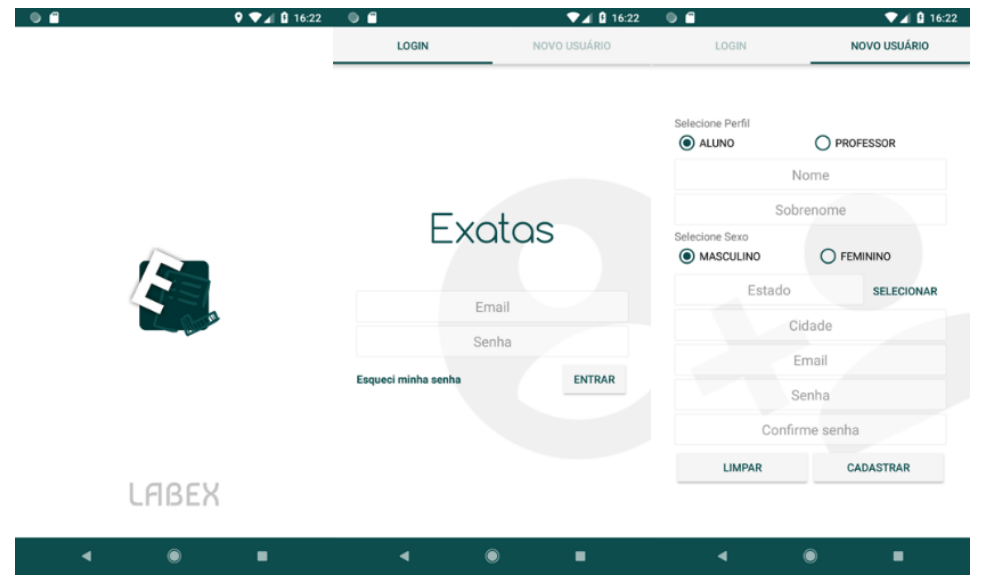

(a)

(b)

(c)

Figura 2 - Autenticação. (a) Tela de apresentação. (b) Tela de login. (c) Tela de cadastro de usuário.

Ao realizar sua autenticação, o usuário é direcionado a tela de início da aplicação. Nesta tela, conforme a Figura 3 (a), são listadas as disciplinas disponíveis, e também é possível acessar o menu lateral de navegação, vide Figura 3 (b). Este permite ao usuário voltar rapidamente ao início como também acessar o seu desempenho, configurações e informações do aplicativo.

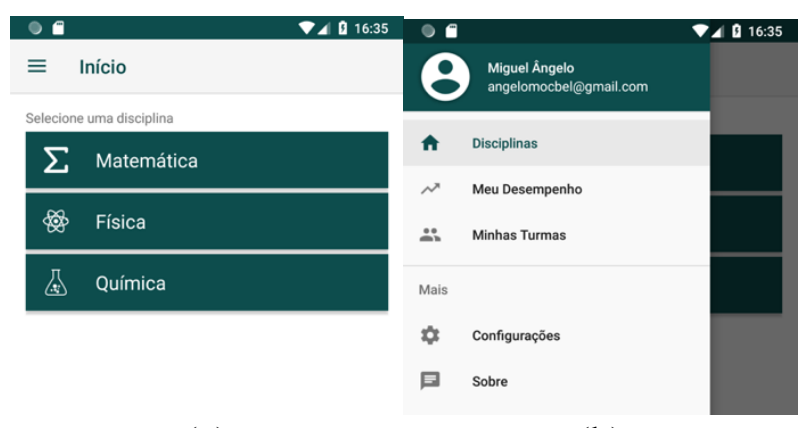

(a)

(b)

Figura 3 - Tela de início. (a) Tela de disciplinas. (b) Menu lateral de navegação.

Ao clicar sobre uma disciplina, por exemplo matemática, é exibido ao usuário a lista de assuntos da disciplina escolhida, vide a Figura 4 (a). A lista de assuntos é organizada por ano escolar, isto é, primeiro são apresentados os assuntos do primeiro ano, depois do segundo e por último do terceiro ano. Ainda nesta tela, é possível filtrar os assuntos por ano escolar clicando em um ícone na barra superior (Figura 4 (b)), assim como, realizar busca por nome a partir do ícone de lupa, conforme Figura 4 (c). Quando o usuário seleciona um assunto, o mesmo é direcionado a uma tela que contém três abas. Cada aba permite o acesso a diferentes materiais de estudo em um mesmo ambiente de aprendizagem, isto é, conteúdo teórico ${ }^{9}$, exercícios ${ }^{10}$ e videoaulas ${ }^{11}$.

\footnotetext{
' https://www.soq.com.br/, https://www.somatematica.com.br/ e https://www.sofisica.com.br/

${ }^{10}$ Questões de vestibulares e concursos realizados no Brasil

"Vídeos produzidos pelos autores em conjunto com os membros do LABEX e também produzidos por colaboradores externos
} 
VII Congresso Brasileiro de Informática na Educação (CBIE 2018)

Anais dos Workshops do VII Congresso Brasileiro de Informática na Educação (WCBIE 2018)

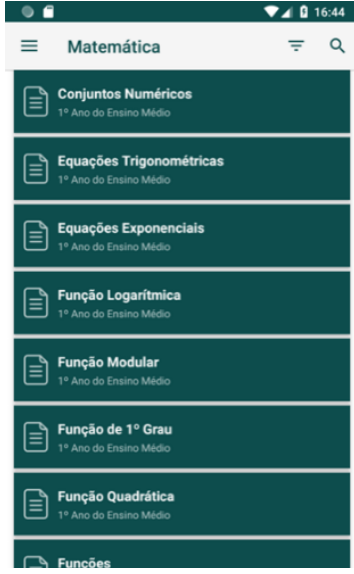

(a)

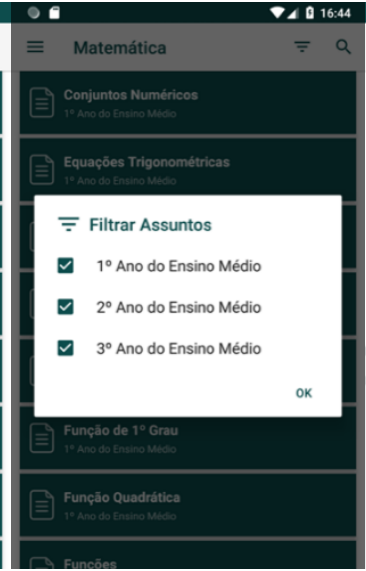

(b)

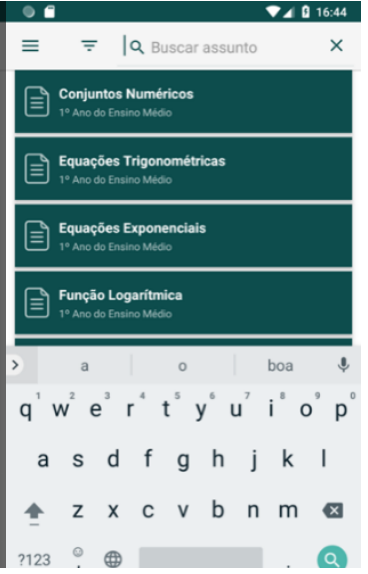

(c)

Figura 4 - Lista de assuntos. (a) Tela de assuntos de uma disciplina. (b) Filtro de assuntos por ano escolar. (c) Busca por nome de assunto.

Na Figura 5 (a), a aba conteúdo carrega o material do assunto selecionado, nesta tela é possível navegar e aplicar zoom sobre o texto. Já na Figura 5 (b), a aba questões permite ao usuário iniciar uma lista de exercícios relacionados a partir do botão de play, como também permite definir o número de questões da lista a partir do botão de configuração que é representado pela imagem de uma engrenagem. Na Figura 5 (c), a aba vídeos carrega as videoaulas disponíveis do assunto abordado.

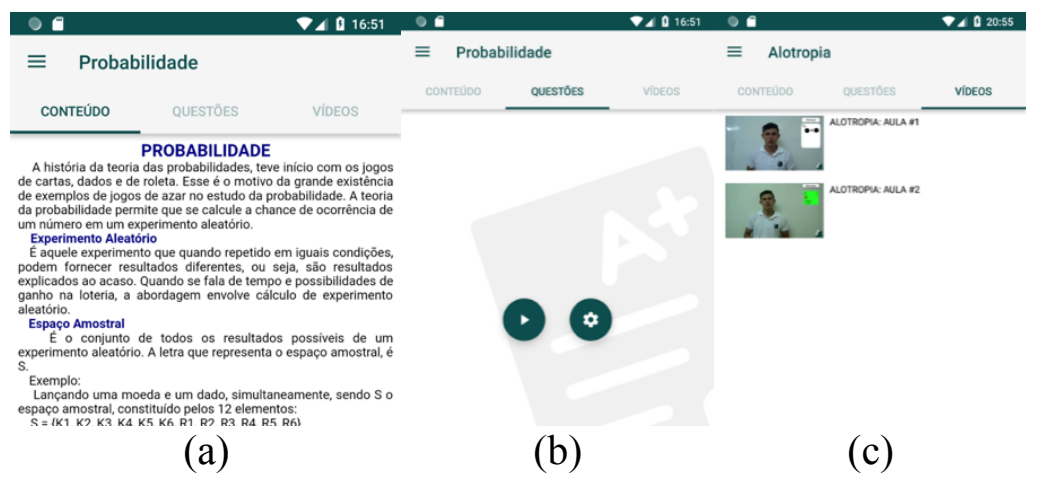

Figura 5 - Tela de assunto. (a) Aba de conteúdo. (b) Aba de questões. (c) Aba de vídeos.

Ao clicar no botão play, o software busca, de forma aleatória, no servidor de banco de dados uma lista de questões. A Figura 6 (a) ilustra a tela de lista de exercícios carregada. Nesta tela, é possível navegar entre as questões a partir dos botões flutuantes localizados na parte inferior e a partir do menu lateral direito, vide Figura 6 (b), além disso, é possível resolver as questões selecionando uma das opções e submetendo a resposta clicando no botão flutuante centralizado. Ao resolver uma questão, o aplicativo retorna ao usuário se o mesmo acertou ou errou, conforme a Figura 6 (c).

Ainda na tela de exercícios, um ícone é mantido ativo na parte superior quando um vídeo de resolução ${ }^{12}$ está disponível para a questão atual (Figura 6 (c)).

${ }_{12}$ Vídeos produzidos pelos autores em conjunto com os membros do LABEX e também produzidos por colaboradores externos. Esses vídeos foram produzidos com o intuito de auxiliar o aluno durante seu 
VII Congresso Brasileiro de Informática na Educação (CBIE 2018)

Anais dos Workshops do VII Congresso Brasileiro de Informática na Educação (WCBIE 2018)

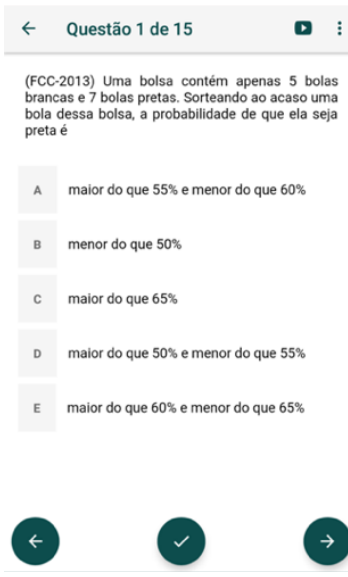

(a)

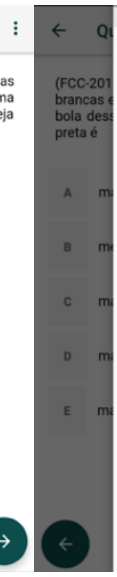

$\leftarrow$ ansoris

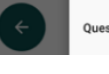

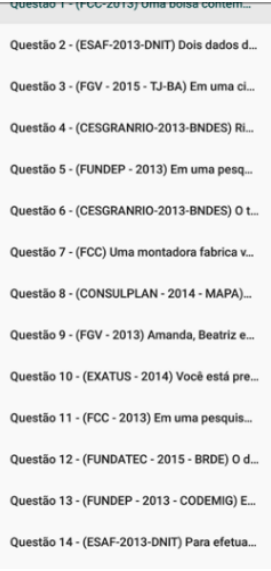

(b)

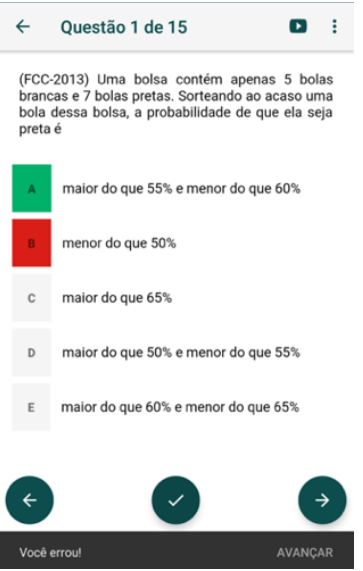

(c)

Figura 6 - Tela de exercícios. (a) Questão em exibição. (b) Acesso rápido a questões. (c) Apresentação da resposta do usuário e resposta correta.

Ao clicar nesse ícone, um vídeo com a explicação da resolução da questão é iniciado, vide Figura 7 (a). Ao finalizar uma lista de exercícios, um gráfico de desempenho é exibido ao usuário com os números de acertos, erros e questões em branco, conforme Figura 7 (b).

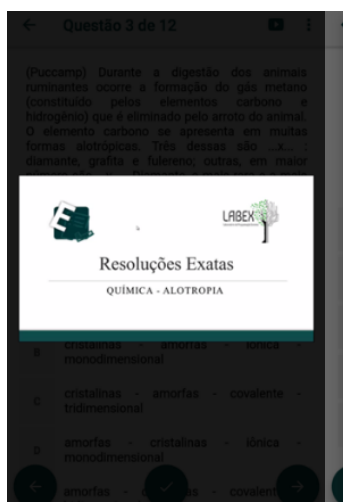

(a)

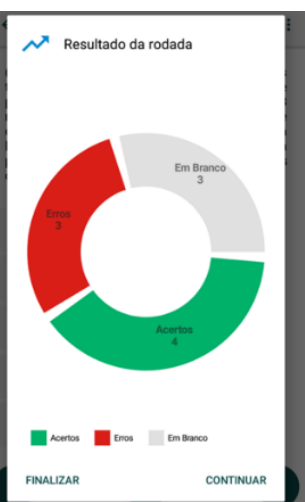

(b)

Figura 7 - Telas do Exatas. (a) Apresentação de vídeos-resolução de uma questão. (b) Apresentação do resultado de uma lista de exercício.

Além de exibir o resultado da lista de questões, acertos e erros são contabilizados para formar o desempenho geral do usuário nas disciplinas de matemática, física e química. Esse desempenho pode ser consultado a partir do menu lateral da tela de inicio do Exatas. Na Figura 8 (a), o desempenho do usuário é organizado por disciplina e é formado pelos percentuais de acertos e erros. É possível ver o desempenho em uma disciplina de forma mais detalhada a partir do botão detalhes, o qual direciona o usuário para uma tela com duas abas. Uma aba exibe em formato gráfico os percentuais organizados por ano escolar (Figura 8 (b)), enquanto a outra aba exibe os números em formato textual (Figura $8(\mathrm{c})$ ).

aprendizado, isto é, caso o aluno tenha dificuldades para resolver determinado exercício a plataforma apresenta um vídeo produzido que explica como resolver tal problema. Desta forma, no Exatas para cada exercício disponibilizado deve-se existir a produção de um vídeo explicativo. 
VII Congresso Brasileiro de Informática na Educação (CBIE 2018)

Anais dos Workshops do VII Congresso Brasileiro de Informática na Educação (WCBIE 2018)

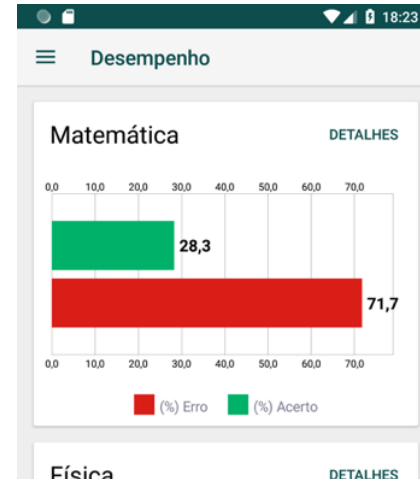

(a)

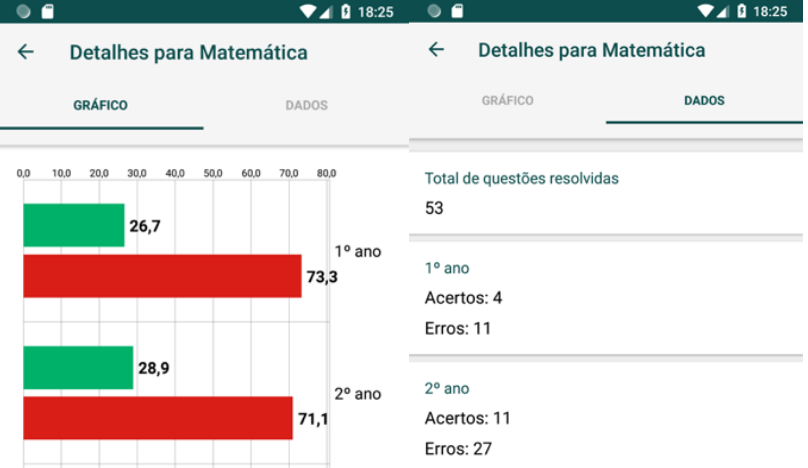

(b) (c)

Figura 8 - Telas de desempenho. (a) Gráficos de desempenho geral por disciplina. (b) Gráfico de desempenho por ano escolar. (c) Números de desempenho por ano escolar.

\section{Considerações finais}

Neste trabalho foi apresentado o software Exatas, que consiste em uma ferramenta que apresenta de forma organizada, conteúdos teóricos, listas de exercícios, desempenho e videoaulas explicativas. Para validação, o software avaliado por 47 alunos dos cursos de graduação em pedagogia e matemática. Durante a avaliação, foram realizadas cinco perguntas de múltipla escolha, onde o usuário era estimulado a responder optando entre as seguintes opções baseadas na escala de Likert [Backer 1995]: concordo completamente, concordo, discordo, discordo completamente e não sei. Para simplificação da apresentação dos resultados, nesta seção são apresentados de forma conjunta os resultados de concordo completamente e concordo, assim como, de discordo e discordo completamente.

A Tabela 1 apresenta os resultados obtidos após a avaliação e estão organizados em identificação da pergunta (P\#), pergunta realizada, e o percentual de cada resposta do usuário, onde (I) representa as respostas concordo e concordo completamente somadas, (II) representa as respostas discordo e discordo completamente também agregadas, e por fim, (III) representa a opção não sei.

A partir da Tabela 1 podemos concluir que mais de 97\% dos entrevistados concordam que o Exatas é fácil de usar, possui interface agradável e sua apresentação é clara e consistente. Assim como, mais de $87 \%$ e $93 \%$ dos entrevistados concordam que o software satisfaz o que é proposto e concordam em indicar o mesmo para outras pessoas, respectivamente. Em consonância com Sena et al. (2014) os resultados obtidos provam que a junção das funcionalidades de conteúdo textual, listas de exercícios e videoaulas em uma única plataforma para plataforma móvel podem ser aceitas de forma positiva pelos usuários quando analisados os itens de P01 até P05.

Além dos testes realizados com as turmas de graduação, o aplicativo também foi avaliado por 990 usuários mantendo uma média de 4,43 estrelas de um total de 5, ou seja, $85,05 \%$ dos usuários classificaram o aplicativo com 4 ou 5 estrelas. Além disso, o software já foi instalado por mais de 212 mil usuários a partir da loja Google Play, conforme dados do Google Play Console [Google 2018]. 
VII Congresso Brasileiro de Informática na Educação (CBIE 2018)

Anais dos Workshops do VII Congresso Brasileiro de Informática na Educação (WCBIE 2018)

Tabela 1 - Resultado da avaliação via formulário aplicados a alunos dos cursos de pedagogia e matemática

\begin{tabular}{ccccc}
\hline P\# & Pergunta & I & II & III \\
\hline P01 & O aplicativo é fácil de usar? & $97,87 \%$ & $0 \%$ & $2,13 \%$ \\
P02 & $\begin{array}{c}\text { O aplicativo apresenta uma interface } \\
\text { gráfica agradável e legível? }\end{array}$ & $97,87 \%$ & $0 \%$ & $2,13 \%$ \\
& $\begin{array}{c}\text { O conteúdo textual está claro e } \\
\text { P03 }\end{array}$ & $97,87 \%$ & $0 \%$ & $2,13 \%$ \\
P04 & O aplicativo satisfaz o que é proposto? & $87,24 \%$ & $10,64 \%$ & $2,13 \%$ \\
P05 & Você indicaria o aplicativo para um amigo & $93,62 \%$ & $0 \%$ & $6,38 \%$ \\
\hline
\end{tabular}

Para projetos futuros, espera-se implementar a área do professor, onde o mesmo poderá criar turmas e simulados, assim como, poderá avaliar e acompanhar o desempenho individual dos alunos a partir das resoluções dos simulados. Na parte do aluno, espera-se implementar uma área, onde o aluno poderá efetuar sua inscrição em turmas e simulados criados pelos seus professores. Por fim e com a evolução deste software, espera-se auxiliar na consolidação do uso do m-learning na aprendizagem das disciplinas de matemática, física e química.

\section{Referências}

Arantes, H. and Seabra, R. (2016) "TME: Aplicativo M-Learning para o Estudo de Conceitos Matemáticos com Ênfase no ENEM", In: Brazilian Symposium on Computers in Education (Simpósio Brasileiro de Informática na Educação-SBIE), p. 1.

Backer, P. (1995), Gestão ambiental: a administração verde, Qualitymark, pages 248.

Crompton, H. (2013) "A Historical Overview of M-learning. Toward Learner- Centered Education”, In: Z. Berge \& L. Muilenburg (Eds.) Handbook of Mobile Learning, New York: Routledge, pages 3-14.

Farias, P. D., Moura, M. P. C., Cantão, V. A. and Farias, F. S. (2017) "Software Educativo para Auxílio no Aprendizado de Química", In: Anais do II Congresso Nacional de Pesquisa e Ensino de Ciências (CONAPESC), p. 1.

Google (2018) "Google Play Console", https://play.google.com/apps/publish/, September.

Mendes, A., Santana, G. and Junior, E. P. (2017) "O Uso do Software PhEt como ferramenta para o Ensino de Balanceamento de Reação Química", Revista Areté I Revista Amazônica de Ensino de Ciências, 8(16), pages 52-60.

Perez, M. C., Vialli, L. And Lahm, R. A. (2015) “Aplicativos para Tablets e Smartphones no Ensino de Física”, Revista Ciências \& Ideais, 7(1), pages 154-173.

Sena, D. M., Oliveira, E. H., Carvalho, L. S. (2014) "Aplicativos móveis para o aprendizado de matemática", In: Brazilian Symposium on Computers in Education (Simpósio Brasileiro de Informática na Educação-SBIE), p. 174. 